\title{
Stable extremely-high-damping discrete viscoelastic systems due to negative stiffness elements
}

\author{
Yun-Che Wang \\ Department of Engineering Physics, Engineering Mechanics Program, University of Wisconsin-Madison, \\ 147 Engineering Research Building, 1500 Engineering Drive, Madison, Wisconsin 53706-1687 \\ Roderic S. Lakes ${ }^{\text {a) }}$ \\ Department of Engineering Physics, Engineering Mechanics Program, Biomedical Engineering Department, \\ Materials Science Program and Rheology Research Center, University of Wisconsin-Madison, \\ 147 Engineering Research Building, 1500 Engineering Drive, Madison, Wisconsin 53706-1687
}

(Received 19 December 2003; accepted 7 April 2004; published online 12 May 2004)

\begin{abstract}
Systems with negative stiffness constituents can have extreme material properties greatly exceeding those of either constituent. We show that a discrete system with a viscoelastic damping element and a negative stiffness element can be made with overall viscoelastic damping orders of magnitude higher than that of any constituent, or of the system with all elements of positive stiffness. The product of stiffness and damping, important for vibration damping, is also enhanced by orders of magnitude. We show this system is unconditionally stable in the high damping regime. The singularity in damping can be made arbitrarily close to the stability boundary. () 2004 American Institute of Physics. [DOI: 10.1063/1.1759064]
\end{abstract}

In ordinary heterogeneous solids, stiffnesses of each phase are positive, and the composite properties cannot exceed the properties of either constituent. Negative stiffness constituents can give rise to extreme properties exceeding those of either constituent as shown theoretically ${ }^{1}$ and experimentally in a buckled lumped system ${ }^{2}$ and in a distributed composite system ${ }^{3}$ containing a dilute concentration of inclusions. In the composite, negative stiffness was achieved by inclusions in the vicinity of a ferroelastic phase transformation. In addition to unusual mechanical properties, anomalies in coupled field responses, such as thermal expansion, piezoelectricity, pyroelectricity, or electric permittivity, have been analyzed. ${ }^{4}$ The causal mechanism for the phenomena is the balance between the contributions from negative stiffness and positive stiffness phases. This gives rise to amplified motion at the interface between constituents. Although response curves resemble resonance, the causal mechanism does not entail any inertial terms, in contrast to resonant effects in which extreme characteristics can be seen with a dilute concentration of heterogeneities ${ }^{5}$ and in contrast to dielectric systems which attain negative permittivity near a resonance. $^{6}$

Negative stiffness and negative Poisson's ratio are distinct. Negative stiffness entails a reversed directional relationship between load and deformation so that an imposed deformation causes an assisting force rather than a restoring force from the material. Poisson's ratio, denoted as $\nu$, is defined as the negative lateral strain of a stressed body divided by its longitudinal strain. Positive definiteness of strain energy, for stable isotropic elastic solids implies $-1<\nu<0.5$. Stable foams with negative Poisson's ratio and positive stiffness with $\nu=-0.8^{7,8}$ have been made. ${ }^{9}$

Negative stiffness can be achieved in prestressed elastic

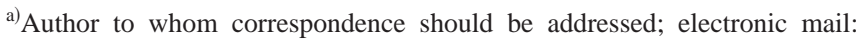
lakes@engr.wisc.edu
}

systems including tubes or structures following buckling, in preloaded lattices, and in ferroelastic materials just below their phase transformation temperature. Negative stiffness by itself usually entails instability. A bulk elastic solid of negative stiffness is unstable, but if all of its boundaries are fully constrained in displacement, it can be stable, provided the shear modulus $(G)$ and Poisson's ratio $(\nu)$ satisfy $G>0$ and $-\infty<\nu<0.5$ or $1<\nu<\infty .{ }^{10}$ Since Young's modulus is $E$ $=2 G(1+\nu)$ for isotropic homogeneous elastic materials, the Young's modulus can be negative for constrained solids. The criterion of strong ellipticity ${ }^{11}$ (modulus tensor $C_{i j k l}$ obeys $C_{i j k l} a_{i} b_{j} a_{k} b_{l}>0$ for any nonzero $a_{i}$ and $b_{j}$, which entails real wave speeds) enforces $G>0$ and $\nu<0.5$ or $\nu>1$, which allows negative Young's and bulk moduli. If strong ellipticity is violated, bands may form in the material, as is observed in ferroelastic solids below a critical transformation temperature. Reuss-type (series) elastic composites with negative stiffness inclusions are unstable. ${ }^{12}$ A discrete model containing multiple prestressed elastic elements ${ }^{13}$ and a viscous element can display extreme overall stiffness; it is dynamically metastable (has a long divergence time) in this regime.

The conditions under which systems with negative stiffness constituents are stable, metastable, or unstable remain to be determined. The experimental demonstrations of extreme behavior in the composite ${ }^{3}$ were done via tuning processes; therefore, the results are consistent with either metastability or stability. The lumped buckled tube system ${ }^{2}$ was externally constrained and would be unstable as a free element.

Here we show that a discrete system with a viscoelastic damping element and a negative stiffness element can be made with overall viscoelastic damping orders of magnitude higher than that of any constituent or of the system with all elements of positive stiffness. We show that this system is unconditionally stable in the high damping regime.

The energy approach to stability analysis is suitable for 


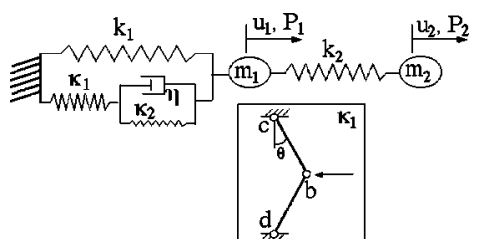

FIG. 1. Linearized discrete viscoelastic system which exhibits extreme high damping due to negative $\kappa_{1}$. Negative $\kappa_{1}$ can be achieved from snapthrough of the elements $b c$ and $b d$ in the inset diagram near $\theta=0^{\circ}$.

conservative, e.g., elastic problems, but not for viscoelastic ones. Therefore we make use of the Liapunov indirect theorem, equivalent to the Routh-Hurwitz method. ${ }^{14}$ If the Jacobian matrix, $\mathbf{J}=\partial \mathbf{M}^{-1} \mathbf{X} /\left.\partial \mathbf{x}\right|_{\mathbf{x}=\mathbf{x}_{e}}$, contains eigenvalues with no positive real parts, then the dynamical system is stable. Here $\mathbf{M}$ is a matrix which may contain masses or moments of inertia (if rotational degrees of freedom present) or viscosity, in the form of a state-space representation, $\mathbf{x}$ is the vector of state-space variables containing generalized coordinates and generalized momenta, and $\mathbf{x}_{e}$ is $\mathbf{x}$ evaluated at a specified equilibrium point. $\mathbf{X}$ is a column vector function containing generalized forces and generalized momenta. $\mathbf{X}$ and $\mathbf{x}$ are constructed either from the Hamiltonian of the system, or Newton's second law and by reducing equations of motion to a system of first-order differential equations. This is illustrated in Eq. (4). The stability of a dynamical system with all zero real parts in its eigenvalues is weak albeit stable. ${ }^{15}$ When all eigenvalues have negative real parts, the degree of stability can be understood as the distance between the imaginary axis and the closest eigenvalue to it. ${ }^{16}$ Furthermore the eigenvalue $(\lambda)$ which has the smallest $|\operatorname{Re}(\lambda)|$ is responsible for the rate of decay [for $\operatorname{Re}(\lambda)<0]$ or growth [for $\operatorname{Re}(\lambda)>0$ ] of amplitudes of the system's normal modes.

Figure 1 shows a linearized discrete system in which negative $\kappa_{1}$ is achieved via the snap through effect illustrated in the inset. That system exhibits geometrical nonlinearity, however for stability analysis it is expedient to linearize the system about the salient equilibrium point $\theta=0^{\circ} . P$ 's are force, $f$ is force in the $\kappa_{2}-\eta$ element, $u$ 's are displacement, $\eta$ is viscosity, and $k$ 's and $\kappa$ 's are spring constants. A lattice can be constructed of such elements.

The governing equations of the system are as follows, with $P_{1}=0$ throughout the analysis,

$$
\begin{aligned}
& {\left[\begin{array}{cc}
m_{1} & 0 \\
0 & m_{2}
\end{array}\right]\left(\begin{array}{l}
\ddot{u}_{1} \\
\ddot{u}_{2}
\end{array}\right)+\left[\begin{array}{cc}
k_{1}+k_{2} & -k_{2} \\
-k_{2} & k_{2}
\end{array}\right]\left(\begin{array}{l}
u_{1} \\
u_{2}
\end{array}\right)+\left(\begin{array}{l}
f \\
0
\end{array}\right)=\left(\begin{array}{c}
0 \\
P_{2}
\end{array}\right)} \\
& f+\frac{\eta}{\kappa_{1}+\kappa_{2}} \dot{f}=\frac{\kappa_{1} \kappa_{2}}{\kappa_{1}+\kappa_{2}} u_{1}+\frac{\kappa_{1} \eta}{\kappa_{1}+\kappa_{2}} \dot{u}_{1} .
\end{aligned}
$$

Equation (1) is the equation of motion and Eq. (2) is the constitutive equation for the damped system. The viscoelastic properties are computed in the frequency domain with the aid of the Fourier transformation. The transformed Eqs. (1) and (2) become and (2) become
Downloaded 05 Apr 2007 to 128.104.198.190. Redistribution subject to AlP i

$$
\begin{gathered}
{\left[\begin{array}{cc}
-\omega^{2} m_{1}+k_{1}+k_{2}+\frac{\kappa_{1} \kappa_{2}+i \omega \kappa_{1} \eta}{\kappa_{1}+\kappa_{2}+i \omega \eta} & -k_{2} \\
-k_{2} & -\omega^{2} m_{2}+k_{2}
\end{array}\right]} \\
\times\left(\begin{array}{c}
\tilde{u}_{1} \\
\tilde{u}_{2}
\end{array}\right)=\left(\begin{array}{c}
0 \\
\tilde{P}_{2}
\end{array}\right) .
\end{gathered}
$$

Here the tilde denotes the Fourier transformed variables. At a specific $\omega$, the overall dynamic compliance is $\left|\widetilde{u}_{2} / \widetilde{P}_{2}\right|$; we let $\omega=0$, to obtain the properties of the system under quasistatic conditions.

Viscoelastic damping is expressed in terms of the loss tangent $\tan \delta \equiv \operatorname{Im}\left\{k^{*}\right\} / \operatorname{Re}\left\{k^{*}\right\}$ for a discrete spring element with complex force constant $k^{*} ; \delta$ is the phase angle between sinusoidal force and displacement in time. For the system in Fig. 1, $\widetilde{P}_{2} / \tilde{u}_{2}$ takes the role of $k^{*}$ and for a bulk solid, a complex modulus $G^{*}$ takes the role of $k^{*}$. To analytically study behavior of $\tan \delta$, we write $\tan \delta$ explicitly in terms of $\kappa_{1}, \kappa_{2}, \eta$, and $\omega$, assuming as an example $m_{1}=0$, $m_{2}=0, k_{1}=10 \mathrm{kN} / \mathrm{m}$, and $k_{2}=5 \mathrm{kN} / \mathrm{m}$. These values are chosen to illustrate the effect of negative stiffness in achieving a high viscoelastic loss tangent. Stability near the singularity is studied in the following.

To analyze stability in the sense of Routh-Hurwitz, Eqs. (1) and (2) are rewritten in state space:

$$
\left(\begin{array}{c}
\dot{u}_{1} \\
\dot{u}_{2} \\
\dot{v}_{1} \\
\dot{v}_{2} \\
\dot{f}
\end{array}\right)=\mathbf{J}\left(\begin{array}{c}
u_{1} \\
u_{2} \\
v_{1} \\
v_{2} \\
f
\end{array}\right)+\left(\begin{array}{c}
0 \\
0 \\
0 \\
\frac{P_{2}}{m_{2}} \\
0
\end{array}\right),
$$

with $v_{1}=\dot{u}_{1}$, where

$$
\mathbf{J}=\left[\begin{array}{ccccc}
0 & 0 & 1 & 0 & 0 \\
0 & 0 & 0 & 1 & 0 \\
-\frac{k_{1}+k_{2}}{m_{1}} & \frac{k_{2}}{m_{1}} & 0 & 0 & -\frac{1}{m_{1}} \\
\frac{k_{2}}{m_{2}} & -\frac{k_{2}}{m_{2}} & 0 & 0 & 0 \\
\frac{\kappa_{1} \kappa_{2}}{\eta} & 0 & \kappa_{1} & 0 & -\frac{\kappa_{1}+\kappa_{2}}{\eta}
\end{array}\right] .
$$

From Eqs. (1), (2), and (4), we construct the $\mathbf{J}$ matrix, by reducing the order of differential equations of motion. Following the Liapunov indirect stability theorem, the eigenvalues of the $\mathbf{J}$ matrix are investigated with respect to the tuning parameters, $\kappa_{1}$ and $\eta$.

We analyze the linear discrete viscoelastic model, shown in Fig. 1, in which negative stiffness is generated by geometrical nonlinearity as shown in the inset. Parameters are chosen above to illustrate the effect of negative stiffness. For Fig. $2 \eta=0.1 \mathrm{kN} \mathrm{s} / \mathrm{m}$, and for Fig. $3, \eta=0.01$ to $0.3 \mathrm{kN} \mathrm{s} / \mathrm{m}$ and $\kappa_{1}$ is tuned. The driving frequency is $1 \mathrm{rad} / \mathrm{s}(0.16 \mathrm{~Hz})$ at node 2. Small masses $m_{1}=m_{2}=10^{-12} \mathrm{~kg}$ at the nodes facilitate computation by avoiding a singular matrix. It was verified that the masses were sufficiently small that no inertial effects were seen; all results are well in the quasistatic re-

AlP license or copyright, see http://apl.aip.org/apl/copyright.jsp 


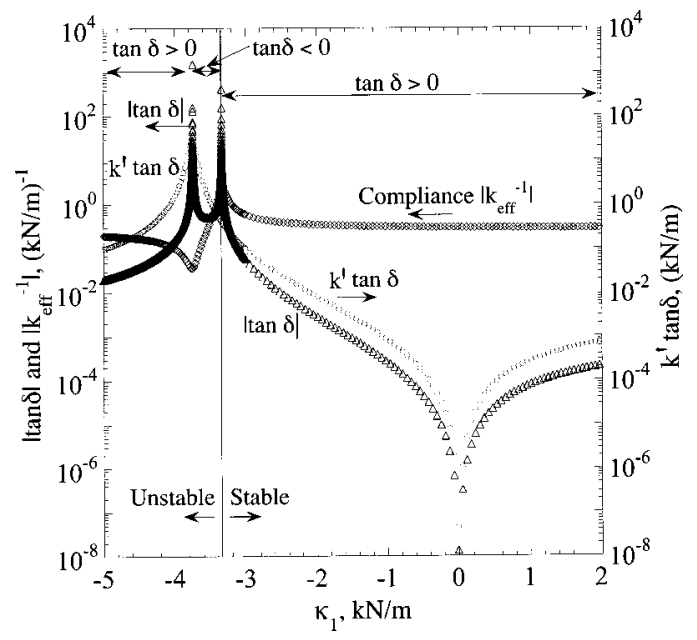

FIG. 2. Stable high damping in a discrete system with preload. Damping $\tan \delta$ (triangles) and compliance (diamonds) vs $\kappa_{1}, k_{1}=10, k_{2}=5, \kappa_{2}=5$ $\mathrm{kN} / \mathrm{m}$. Quasistatic behavior; no inertial terms. $k^{\prime} \tan \delta$ (circles) is a figure of merit for vibration absorption.

Figure 2 shows the overall damping $\tan \delta$ and compliance, versus the value of $\kappa_{1}$, which can be negative. The stability boundary is drawn based on the criterion that the real parts of all eigenvalues must be less than zero for stability. The damping $\tan \delta$ exhibits singularities at $\kappa_{1} \approx-3.34$ and at -3.7 . Also $\tan \delta \rightarrow 0$ when $\kappa_{1} \rightarrow 0$ due to the decoupling of the damper element. The extremely high and low damping are located in the stable regime. Tan $\delta$ becomes negative and then positive as $\kappa_{1}$ decreases in the unstable regime. As seen in Fig. 2 the high damping $\tan \delta$ combined with sufficiently high stiffness $k^{\prime}$ can be achieved so that the product $k^{\prime} \tan \delta$ is enhanced by a factor of more than 528 (about $0.407 \mathrm{kNm}$ at $\kappa_{1}=-3.34$, compared to about 0.00077 at $\kappa_{1}=2$ ). This product is a figure of merit for vibration damping applications.

Stability is governed by eigenvalues of $\mathbf{J}$, with dimensions of inverse time, representing the rate at which a normal

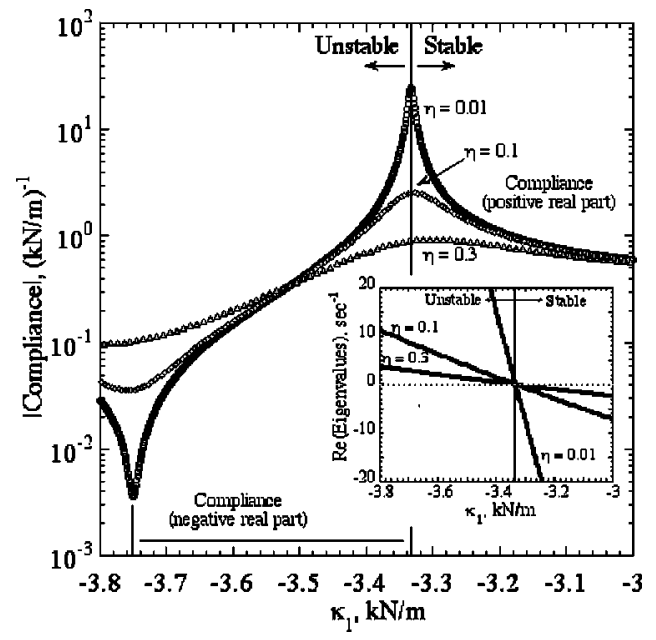

FIG. 3. Effect of viscosity $\eta$ (in units of $\mathrm{kN} \mathrm{s} / \mathrm{m}$ ) on the compliance and on the least stable eigenvalue as $\kappa_{1}$ is varied near the stability boundary (vertical line). Quasistatic behavior; no inertial terms. mode amplitude tends to equilibrium in the stable case, or diverges from equilibrium in the unstable case. The eigenvalues, the least stable of which is shown in Fig. 3, therefore provide a measure of the degree of stability or instability. In the stable regime close to the stability boundary, the overall $\tan \delta$ (about 400 at $\kappa_{1}=-3.34$ ) is about a million times greater than that with all positive stiffness elements (about 0.0002 at $\kappa_{1}=2$ ). Near the boundary on the unstable side, the system may be viewed as dynamically metastable due to the long time constant for divergence in which mass points $m_{1}$ and $m_{2}$ (Fig. 1) move in the same direction. This time constant can be substantially larger than the viscoelastic time constant $\eta / \kappa_{2}=0.02 \mathrm{~s}$.

The stability boundary is aligned with the peak of the effective compliance, as seen in Fig. 3, and the boundary is independent of subresonant (quasistatic) frequency. The peak of the compliance is broader for large viscosity $\eta$. Viscosity does not change the stability boundary, but it does change the degree of instability (stability) in the unstable (stable) regime. When viscosity is sufficiently small, there are singularities in the effective $\tan \delta$. When viscosity approaches zero, one of the singularities approaches the stability boundary.

In summary, the viscoelastic damping of a discrete system can be greatly magnified by use of a negative stiffness element in the system. Such a system can be stable in the Routh-Hurwitz sense with no external constraint. The present results indicate extreme behavior may be expected in the case of unit cells of sufficient complexity. Examples may include superlattices, molecular crystals, or complex intermetallics, as well as composites with nano-structure such as buckled nanotubes or composites with inclusions which undergo phase transformation. Also, the product of stiffness and damping, a figure of merit for vibration absorption, is orders of magnitude greater in the presence of negative stiffness elements.

The authors are grateful for a grant, CMS-0136986, from NSF. We thank W. J. Drugan and R. Carpick for discussions and encouragement.

${ }^{1}$ R. S. Lakes, Phys. Rev. Lett. 86, 2897 (2001).

${ }^{2}$ R. S. Lakes, Philos. Mag. Lett. 81, 95 (2001).

${ }^{3}$ R. S. Lakes, T. Lee, A. Bersie, and Y. C. Wang, Nature (London) 410, 565 (2001).

${ }^{4}$ Y. C. Wang and R. S. Lakes, J. Appl. Phys. 90, 6458 (2001).

${ }^{5}$ N. A. Nicorovici, R. C. McPhedran, and G. W. Milton, Phys. Rev. B 49, 8479 (1994)

${ }^{6}$ D. J. Bergman and D. Stroud, Solid State Phys. 46, 147 (1992).

${ }^{7}$ R. S. Lakes, Science 235, 1038 (1987).

${ }^{8}$ R. S. Lakes, Advanced Materials (Weinheim, Germany, 1993), Vol. 5, pp. 293-296.

${ }^{9}$ G. Milton, J. Mech. Phys. Solids 40, 1105 (1992).

${ }^{10}$ J. H. Bramble and L. E. Payne, Proceedings of the U.S. Fourth National Congress of Applied Mechanics, 1963, pp. 469-473.

${ }^{11}$ J. K. Knowles and E. Sternberg, J. Elast. 8, 329 (1978).

${ }^{12}$ R. S. Lakes and W. J. Drugan, J. Mech. Phys. Solids 50, 979 (2002).

${ }^{13}$ Y. C. Wang and R. S. Lakes, Am. J. Phys. 72, 40 (2004).

${ }^{14}$ L. Meirovitch, Methods of Analytical Dynamics (McGraw-Hill, New York, 1970)

${ }^{15}$ S. H. Strogatz, Nonlinear Dynamics and Chaos (Perseus, Cambridge, MA, 1994).

${ }^{16}$ H. Leipholz, Stability Theory (Wiley, New York, 1987). 